\title{
A survey of staphylococcal infections in group 9 hospitals
}

\author{
JOAN M. READ AND E. YENSON \\ From Group 9 Laboratory, Watford and Watford Maternity Hospital
}

SYNOPSIS This is a three-year survey of staphylococcal infection in the Watford group of hospitals. The organisms are divided into groups according to their pattern of sensitivity to antibiotics. The spread of Staphylococcus pyogenes in a maternity hospital and the measures taken to eradicate it are described.

The incidence of staphylococcal infection and the increasing resistance of this organism to antibiotics has been observed over a number of years in the Watford group of hospitals. Since Staph. pyogenes phage type 80 made its appearance early in 1957, a record has been kept of every case of staphylococcal infection, thus figures for the incidence of different strains of organisms are readily available. (It must be stressed that laboratory figures are dependent on the material sent in for culture, and swabs are not always sent from every minor septic lesion in the hospitals and community.)

In Fig. 1 the staphylococci isolated in the Group 9 Laboratory during the years 1958, 1959, and 1960 have been divided into groups according to their pattern of sensitivity to antibiotics. Only one group was separated on the basis of phage typing and is made up of organisms belonging to type 80 . These organisms were all resistant to penicillin and tetracycline. The main groups are subdivided into numbers of infections among in-patients and outpatients.

This histogram shows the great reduction in numbers of the type 80 staphylococcus achieved during the three years (group A). It also illustrates the increasing number of staphylococci resistant to more than one antibiotic that do not belong to phage type 80 (group B) and resistant to erythromycin (group E). The penicillin-sensitive staphylococcus (group D) remains the most common infecting organism among out-patients. There was a marked all-round reduction of infection during 1960.

WATFORD MATERNITY HOSPITAL Though large numbers of phage type 80 staphylococci were being isolated from all the hospitals, special concern was

Received for publication 25 July 1961. felt over their presence in the Watford Maternity Hospital. Figure 2 is a record of every case of staphylococcal infection occurring in the hospital during the three years. The lesions were mostly of a mild nature, such as skin pustules and sticky eyes, among the infants. Most of the staphylococci isolated from mothers were from high vaginal swabs.
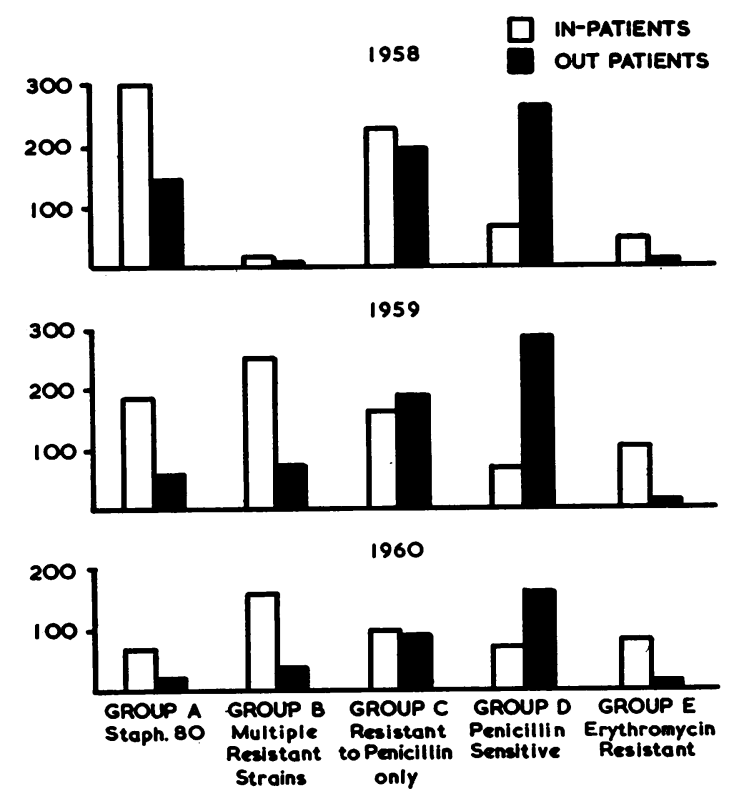

FIG. 1. Staphylococcal infections in Group 9 hospitals, 1958-60

There was a small number of breast abscesses among both mothers and babies.

In September 1958 the staphylococcal content of 


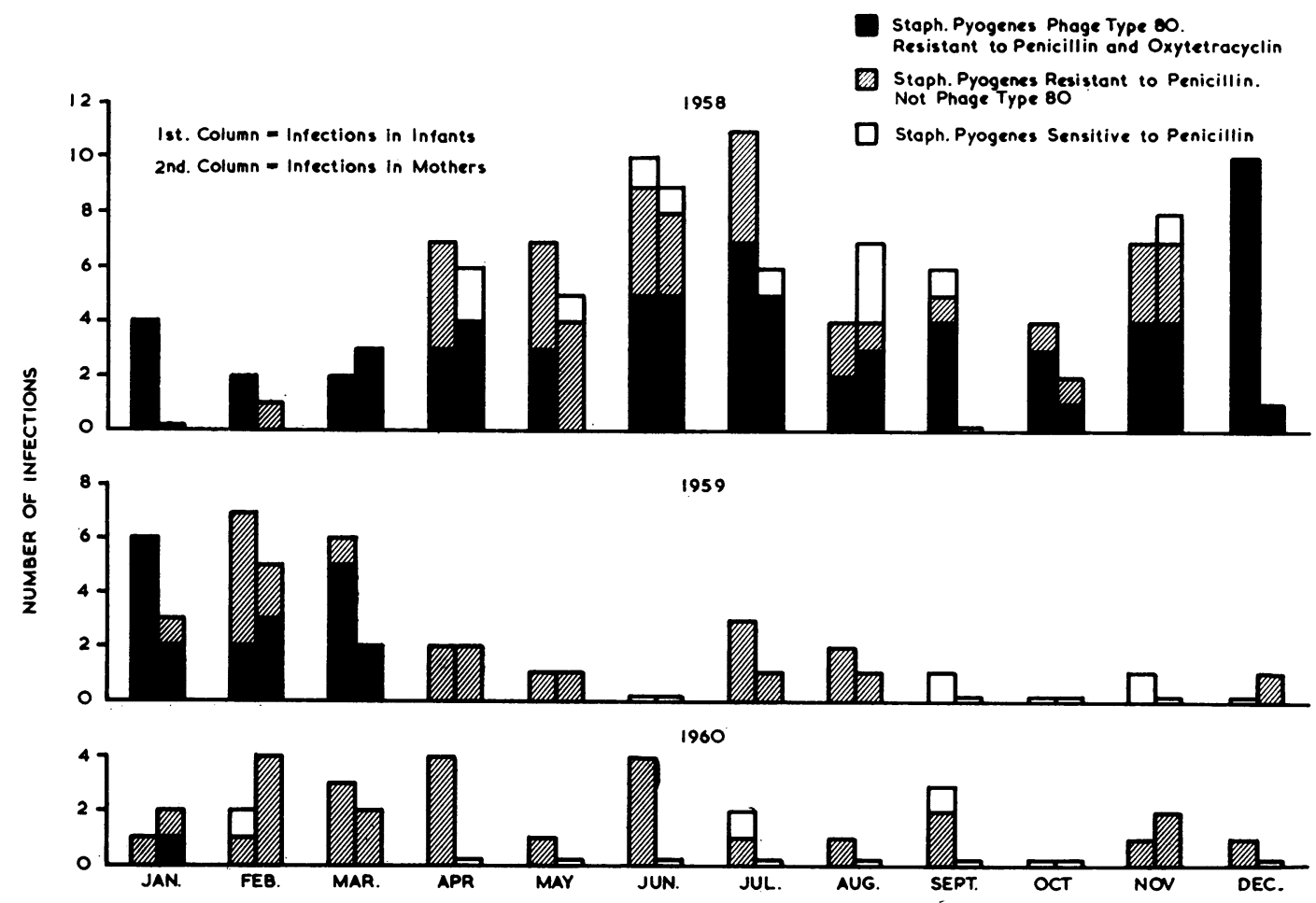

FIG. 2. Staphylococcal infections in Watford Maternity Hospital, 1958-60

the air was examined by exposing six phenolphthalein-phosphate penicillin plates at a time for two hours. An average of two colonies of Staphylococcus aureus belonging to phage type 80 per 10 sq. in. of medium were grown. Repeated swabbing of wrists and noses of nursing staff during these months failed to reveal a carrier.

During January, February, and March 1959 Staph. aureus phage type 80 was still being isolated from patients with septic lesions in the hospital. In March the staphylococcal content of the air was again examined. On this occasion less than one colony of Staph. aureus phage type 80 per 10 sq. in. of medium was grown.

BREAST ABSCESSES During 195845 patients with breast abscesses were treated in the general hospitals. Forty of these abscesses, all of which were in women delivered in the Watford Maternity Hospital, were caused by the phage 80 staphylococcus. The remaining five were in patients delivered at home, and were caused by staphylococci not belonging to phage type 80 . In that year there were 1,200 hospital deliveries and 600 domiciliary deliveries.

The number of breast abscesses treated during
1959 was markedly reduced. The total for the year was twenty-three. Seventeen of these were in patients delivered in the Watford Maternity Hospital and ati were caused by type 80 staphylococci. These a occurred during the first four months of the yeas when there was still infection in the hospital. The other six patients were not confined in the hospita and were infected by staphylococci not belonging to phage type 80 . Since the eradication of the phage 80 staphylococcus from the hospital in April 1959 there have been only two breast abscesses in patients. confined in the hospital, both caused by penicillirro sensitive staphylococci not belonging to phage type 80 . It is realized that there may have been patients with abscesses treated outside the main hospitals from whom pus was never sent to the laborator

Fig. 3 shows the intervals between delivery and the appearance of the maternal breast abscess durine the first five months of 1958 , the most commont interval being four to six weeks (Knight and Nolaㅁ 1959; Wilkinson, 1959; Soltau and Hatcher, 1960ㅇㅇㅇ

SOURCES AND TRANSMISSION OF STAPHYLOCOC $\frac{\rho}{\mathbb{Q}}$ Many workers have written about the virulence of the phage 80 staphylococcus in connexion with newt 


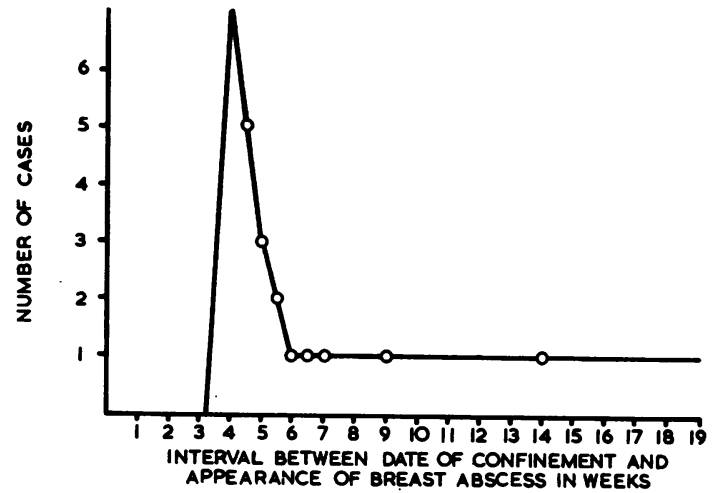

FIG. 3. Interval between confinement and appearance of breast abscess.

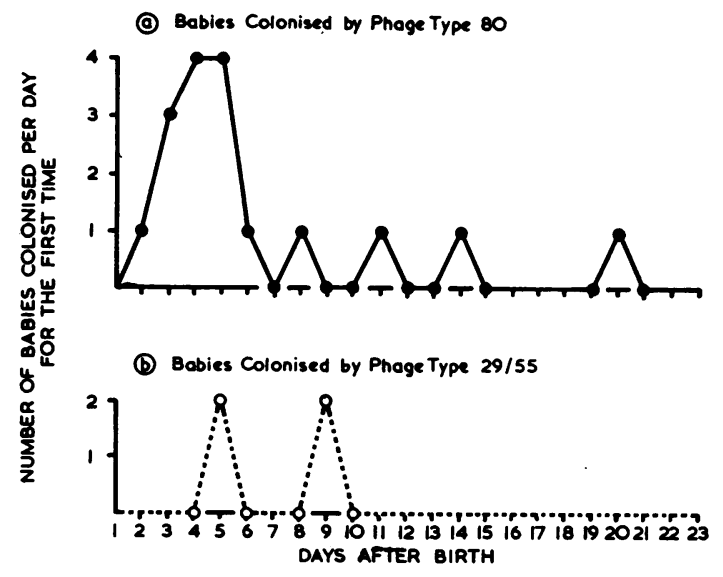

FIG. 4. Nasal colonizaton of infants.

born babies (Gillespie, Pope, and Simpson, 1957; Cook, Parrish, and Shooter, 1958; Barber and Kuper, 1951; Cavanagh, 1960), and Cunliffe (1949, 1957) has shown that colonization of infants' umbilical cords and noses begins in the first days of life.

During January and February 1959 a series of 21 babies had their noses swabbed daily from birth until discharge. All these babies became staphylococcal carriers, though one, who was in hospital 23 days, did not become colonized until the twentieth day. Seventeen $(81 \%)$ of the infants were colonized by phage type 80 staphylococci (Fig. 4a). The remaining four infants were colonized by a less virulent strain, phage type 29/55 (Fig. 4b).

Over the same period 26 mothers' noses were swabbed daily. One mother was admitted carrying a phage type 80 staphylococcus and remained a carrier throughout her stay. Twenty-one carried organisms either sensitive to penicillin, or resistant to penicillin only, not belonging to phage type 80 . Four remained free of staphylococci. No mother was colonized by the predominant hospital strain. Williams, Jevons, Shooter, Hunter, Girling, Griffiths, and Taylor (1959) found that patients who were not carriers on admission acquired resistant strains in hospital more readily than those initially carrying sensitive strains. In our series only four mothers $(15.2 \%)$ were admitted not carrying staphylococci and during their short stay in hospital remained uncolonized.

The original source of Staph. pyogenes of phage type 80 in the Watford Maternity Hospital will never be known. One mother is known to have been a nasal carrier on admission. There may well have been others before her. Though no carrier was found among the staff in 1958, this does not exclude a carrier introducing the organism earlier. Hare and Ridley (1958) and Ridley (1959) have shown that many adults who are nasal carriers of Staph. pyogenes are also skin carriers of this organism, and $22 \%$ can be classed as perineal carriers. Visitors as well as patients may bring organisms into a hospital.

The following three cases were recorded to illustrate the types of patient who can bring infection into a hospital. They were all admitted when the epidemic, viewed in retrospect, was coming under control. Case 3 was one of the last patients in the Watford Maternity Hospital with an infection caused by Staph. pyogenes phage type 80 . Despite her admission there was no further outbreak of infection.

Case 1 In January 1959 Mrs. H. R., a visiting midwife to the hospital, was found to have boils on the buttocks and in the labial region, from which Staph. pyogenes phage type 80 was cultured. There were old scars in the perineal region giving evidence of previous boils.

Case 2 Mrs. J. J. was admitted on 4 January 1959 in premature labour, 14 days after an appendicectomy operation, with a discharging wound from which Staph. pyogenes phage type 80 was isolated.

Case 3 Mrs. N. B., aged 25 years, was admitted to the Watford Maternity Hospital on 8 January 1959 with a Staph. pyogenes (not phage type 80 ) urinary infection. She was in the ward for eight days. She had suffered from widespread eczema from birth. On 16 February 1959, at 38 weeks of pregnancy, she was admitted to a surgical ward with a left breast abscess which was incised and drained. Staph. pyogenes phage type 80 was cultured from the pus. On 15 March 1959, at 41 weeks of pregnancy, the patient was transferred to the labour ward in strong labour. She still had a discharging sinus in the left breast. 


\section{MEASURES TAKEN TO PREVENT} STAPHYLOCOCCAL INFECTION

In 1957 dry sweeping of floors was replaced by suction cleaning followed by wet mopping with a disinfectant. At the same time washable plastic covers for the mattresses and pillows were introduced.

In August and September 1958 all the floors were resurfaced. In December 1958 the wards were closed in rotation for thorough cleaning. This closing and cleaning is now carried out at least once a year.

In January 1959 hexachlorophene powder was introduced for the babies. This was applied to the umbilical area of every newborn baby after the cord had been cleaned with methylated spirit. This was repeated every time the napkin was changed. No cord binders were used. Whenever babies were handled the doctor or nurse wore a clean gown and mask, and the hands were washed with hexachlorophene soap, dried with disposable paper towels, and rubbed with chlorhexidine antiseptic cream. By February 1959 all woollen blankets had been replaced by cellular cotton blankets for both mothers and babies (Schwabacher, Salsbury, and Fincham, 1958), and all the babies' garments were made of cotton. All fabrics were therefore able to stand up to boiling and this made possible the use of sterilized linen and blankets for each mother and her baby. Also in February 1959 hexachlorophene soap was used in all wards and in the theatre but not in the Nurses' Home. In March 1959 canopies placed over sterilizers as 'steam-traps' were removed and more efficient steam outlets installed.

\section{DISCUSSION}

Experience has shown that when virulent organisms are introduced into a hospital, and are not quickly and completely eradicated by antibiotics, crossinfection between patients and between wards is easily established. We have found that the most dangerous patients are those who expectorate. Many of these patients are chronic bronchitics who have received prolonged treatment with many different antibiotics, and we found that the numbers of resistant staphylococci increased during the winter months when these patients were admitted. Another cause for an outbreak of infection was a patient with a colostomy from whose faeces a resistant Staph. pyogenes was cultured (Schwabacher, Salsbury, and Loosemore, 1959). Patients with widespread eczema are easily infected and Hare (1961) stresses the danger of the dissemination of organisms by patients with this condition.
If patients with severe infections are not completely freed from infection while in hospital, they will spread their organisms among the community and cause further infection. These newly infected patients $\overline{0}$ may require hospital treatment and so a vicious 을 circle of spread of infection is set up. In our experi- $\frac{\bar{D}}{\bar{T}}$ ence this cycle can only be broken in the hospitals ${ }_{\propto}$ where strict measures for the eradication of infection can be enforced.

The organism that caused most concern during. the years $1958-60$ was the type 80 staphylococcus. It $\vec{\omega}$ has been encouraging to see how it has disappeared from the community following its eradication from응 the hospitals. The reasons for its decline are not: completely clear and are probably numerous. The following factors are considered to be important:(1) The eradication of the virulent members of the $\nexists$ strain by erythromycin; (2) the use of hexachloro-o phene powder to reduce colonization of the babies'umbilical cords in the Maternity Hospital; (3) the 3 use of hexachlorophene soap in the wards and theatres of all the hospitals; (4) general measures for $\stackrel{ }{5}$ the prevention of cross infection such as werece taken in the Maternity Hospital in 1959; (5) the N isolation and treatment of septic cases; (6) ecological variation in the virulence of the organism (Wilson, 1957).

We should like to thank Mr. MacRae for giving us the opportunity to write this report, and Dr. Schwabacher $\overrightarrow{\vec{t}}$ for giving us access to her records. We are indebted to음 Dr. R. E. O. Williams and Dr. M. Patricia Jevons for phage typing the staphylococci.

\section{REFERENCES}

Barber, Mary, and Kuper, S. W. A. (1951). J. Path. Bact., 63, 65. Cavanagh, Florence (1960). Brit. med. J., 1, 468.

Cook, Josephine, Parrish, J. A., and Shooter, R. A. (1958). Ibid.O $1,74$.

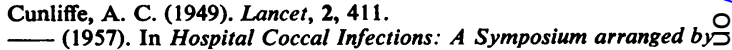
the Association of Clinical Pathologists and the Medical Research Council Committee on Cross-Infection in Hospital, ed. R. E. OTO Williams and R. A. Shooter, p. 1. Gillespie, W. A., Pope, Rosemary C., and Simpson, K. (1957) $\mathrm{N}$
Brit. med.J., 1, 1044.

Hare, R. (1961). Communication Path. Soc. Gt. Brit. and Ireland Jan. 6th.

—, and Ridley, M. (1958). Brit. med. J., 1, 69.

Knight, I. C. S., and Nolan, B. (1959). Ibid., 1, 1224.

Ridley, M. (1959). Ibid., 1, 270.

Schwabacher, Herta, Salsbury, A. J., and Fincham, W. J. (1958) Lancet, 2, 709.

- and Loosemore, T. G. E. (1959). J. clin. Path., 12, 565ף Soltau, D. H. K., and Hatcher, G. W. (1960). Brit. med. J., 1, 1603? Wilkinson, Barbara M. (1959). J. Obstet. Gynec. Brit. Cwlth, 66, 394. Williams, R. E. O., Jevons, M. P., Shooter, R. A., Hunter, C. J. W. $\overline{0}$ Girling, J. A., Griffiths, J. D., and Taylor, G. W. (1959) Brit. med. J., 2, 658

Wilson, G. S. (1957). In Microbial Ecology: Seventh Symposium of the Society for General Microbiology, p. 338. University Press Cambridge. 